\title{
ANÁLISE PROBABILÍSTICA DA ESTABILIDADE DE TENSÃO EM SISTEMAS COMPOSTOS DE GERAÇÃO E TRANSMISSÃO
}

\author{
Anselmo Barbosa Rodrigues* \\ nebulok_99@yahoo.com
}

\author{
Ricardo B. Prada* \\ prada@ele.puc-rio.br
}

\author{
Maria da Guia da Silva ${ }^{\dagger}$ \\ guia@dee.ufma.br
}

*Departamento de Engenharia Elétrica, PUC-Rio, Rua Marquês de São Vicente, 225, CEP 22453-900, Rio de Janeiro, RJ

${ }^{\dagger}$ Departamento de Engenharia Elétrica, UFMA, Av. dos Portugueses, S/N, CEP 65080-040, São Luís, MA

\section{RESUMO}

A instabilidade de tensão geralmente manifesta-se de duas formas: insolubilidade das equações de fluxo de potência e a perda de controlabilidade. Entretanto, o último mecanismo não tem sido considerado na Análise Probabilística da Estabilidade de Tensão (APET). O objetivo deste artigo é apresentar um método para incluir a perda de controlabilidade e a insolubilidade na APET. O método proposto se baseia na combinação de três técnicas: Método Monte Carlo (MMC), Fluxo de Potência Ótimo (FPO) não-linear e o Método da Matriz D' (MMD). Os resultados dos testes demonstraram que a perda de controlabilidade é um mecanismo importante na APET.

PALAVRAS-CHAVE: Estabilidade de Tensão, Método Monte Carlo, Fluxo de Potência Ótimo, Confiabilidade Composta, Métodos Probabilísticos.

\section{ABSTRACT}

Probabilistic Assessment of Voltage Stability in Composite Generation and Transmission Systems

The voltage instability is usually characterized by two mechanisms: unsolvability of the power flow equations and controllability loss. However, the last mechanism has not been

Artigo submetido em 19/11/2009 (Id.: 01081)

Revisado em 28/01/2010, 03/03/2010

Aceito sob recomendação do Editor Associado Prof. Antonio Carlos Zambroni de Souza considered in the voltage stability probabilistic assessment. The objective of this paper is to present a method to include controllability loss and unsolvability in the voltage stability probabilistic assessment. The proposed method is based on the combination of three techniques: Monte Carlo Simulation Method, Nonlinear Optimal Power Flow and D' Matrix Method. The test results demonstrated that the controllability loss is an important mechanism in the voltage stability probabilistic assessment.

KEYWORDS: Voltage Stability, Monte Carlo Method, Optimal Power Flow, Composite Reliability, Probabilistic Methods.

\section{INTRODUÇÃO}

Atualmente, os sistemas de energia elétrica estão operando próximos de seus limites. Esta condição de operação é causada pelos seguintes fatores: crescimento natural da demanda de energia elétrica e adiamento da expansão da transmissão devido a restrições de roteamento e reduções no orçamento do setor elétrico resultantes de dificuldades econômicas. A operação de linhas de transmissão altamente carregadas tem dado origem a problemas de instabilidade de tensão nas redes elétricas. A estabilidade de tensão é definida como a capacidade de um sistema de energia elétrica para manter as tensões controláveis em todas as barras após a ocorrência de distúrbios tais como: flutuações de carga e contingências nos equipamentos do sistema (Kundur 1994; Ajjarapu 2006). Es- 
tados de instabilidade de tensão são causados principalmente por dois mecanismos:

i) Ausência de Solubilidade (Overbye 1994; Granville, Mello, Melo 1996): após a ocorrência de um distúrbio as equações de fluxo de potência não têm solução real devido à violação no limite de máxima transferência de potência para as barras de carga. A distância entre o ponto de carregamento atual e o ponto de carregamento máximo é denominada Margem de Estabilidade de Tensão (MET).

ii) Perda de Controlabilidade (Kundur 1994; Prada et al. 2002): após a ocorrência de um distúrbio, as ações de controle, usadas para corrigir o perfil de tensão, têm efeito oposto ao esperado. Por exemplo, a redução da tensão em uma barra após o chaveamento de um banco de capacitores. Este efeito é devido ao valor negativo da relação de sensibilidade QV na barra onde o capacitor foi chaveado.

É possível identificar com precisão se um estado do sistema tem problemas de instabilidade de tensão, causados pela perda de controlabilidade ou insolubilidade, usando-se uma das seguintes técnicas: Análise Modal (Gao, Morison \& Kundur 1992), Fluxo de Potência Continuado (Ajjarapu \& Christy 1992), FPO (Granville, Mello \& Melo 1996) e MMD (Prada et al. 2002). Esta identificação pode ser realizada porque os mecanismos de instabilidade de tensão estão associados com a existência de soluções reais para as equações de fluxo de potência e com as relações de sensibilidade entre variáveis de controle e estado. Entretanto, não é possível prever o estado de operação do sistema. Esta restrição é causada pelo comportamento estocástico das flutuações de carga e falhas nos equipamentos. Consequentemente, há uma incerteza com relação à ocorrência de estados de instabilidade de tensão. Portanto, é importante que a análise de estabilidade de tensão também reconheça as incertezas associadas com a natureza aleatória dos distúrbios do sistema. Sob este ponto de vista, os métodos mais adequados para modelar incertezas, associadas com distúrbios no sistema, são os métodos probabilísticos. A principal vantagem dos métodos probabilísticos é a sua capacidade para combinar severidade e probabilidade para expressar de forma realística o risco do sistema (Billinton \& Allan 1996; Billinton \& Li 1994). Além disso, vários distúrbios causados por problemas de instabilidade de tensão têm sido relatados na literatura (Ajjarapu 2006; Prada et al. 2002). Estes eventos motivaram o desenvolvimento de ferramentas para quantificar o Risco de Instabilidade de Tensão (RIT). Este interesse em calcular o RIT resultou em diversos artigos sobre a APET (Melo, Mello \& Granville 1997; Billinton \& Aboreshaid 1998; Li et al. 1998; Aboreshaid \& Billinton 1999; Leite da Silva et al. 2000; Wan, McCalley \& Vittal 2000; Huang \& Nair 2002). Nestas publicações, a APET foi realizada usando duas técnicas: o Método de Enumeração de Estados e o MMC. Estas técnicas foram usadas para modelar as seguintes incertezas associadas com os distúrbios causadores da instabilidade de tensão: erros de previsão do pico de carga e indisponibilidades dos equipamentos (falhas em geradores, linhas e transformadores). A modelagem destas incertezas possibilitou a estimação de índices probabilísticos tais como: RIT das barras e do sistema, MET das barras e do sistema e o corte de carga esperado devido a problemas de instabilidade de tensão. Estes índices foram estimados considerando-se dois critérios para identificar os estados instáveis: ausência de solubilidade ou violação no limite da MET. Em outras palavras, estados instáveis causados pela perda de controlabilidade não foram considerados na APET. Apesar disso, problemas de perda de controlabilidade foram relatados em diversos incidentes de instabilidade de tensão, tais como o distúrbio do sistema brasileiro Sul-Sudeste ocorrido em 24 de abril de 1997 (Prada et al. 2002). Desta forma, o principal objetivo deste artigo é modelar a perda de controlabilidade e a ausência de solubilidade na APET. Este modelo é baseado na combinação de três técnicas:

i) MMC (Billinton \& Li 1994): para modelar incertezas associadas com erros de previsão de carga e indisponibilidade dos equipamentos,

ii) MMD (Prada et al. 2002): para incluir problemas de perda de controlabilidade nos índices probabilísticos associados com estados instáveis,

iii) FPO não-linear via Método de Pontos-Interiores (Granville, Mello \& Melo 1996): para avaliar estados instáveis associados com a ausência de solubilidade.

Neste ponto é importante mencionar que os estados instáveis causados pela perda de controlabilidade também podem ser identificados usando-se a Análise Modal (Gao, Morison \& Kundur 1992). Entretanto, a Análise Modal tem um custo computacional superior aquele associado com o MMD. Esta desvantagem da Análise Modal deve-se à necessidade de calcular autovalores, autovetores e fatores de participação para avaliar a estabilidade de tensão de uma barra do sistema. Por outro lado, o MMD exige somente soluções progressivas/regressivas, com os fatores LU da matriz Jacobiana, para realizar uma análise de estabilidade de tensão nodal. O custo computacional é um aspecto crítico na APET visto que se deve avaliar um grande número de estados para estimar os índices com uma precisão aceitável.

A combinação das três técnicas citadas foi usada para gerar os seguintes índices probabilísticos: RIT, probabilidade dos estados de robustez (Fotuhi-Firuzabad \& Billinton 1997; Leite da Silva et al. 2008) e porcentagens das incertezas e dos mecanismos de instabilidade com relação ao RIT. Estes 
índices foram calculados para três sistemas-teste: dois sistemas equivalentes obtidos a partir do Sistema Interligado Nacional (Alves 2007) e uma versão modificada do sistema IEEE para estudos de confiabilidade (Bertoldi, Salvaderi \& Scalcino 1988). Os resultados obtidos com os três sistemasteste demonstraram que a perda de controlabilidade tem um impacto significante nos índices probabilísticos associados com estados instáveis.

\section{DESCRIÇÃO DO MÉTODO}

A análise de estabilidade de tensão de uma rede elétrica é uma função de parâmetros tais como: nível de carga, padrões de geração e topologia da rede. Estes parâmetros estão associados com incertezas associadas com erros de previsão de carga e falhas dos componentes. Neste artigo, as incertezas associadas com parâmetros são incluídas na APET através do MMC. O erro de previsão de carga é modelado usando uma distribuição normal (Billinton \& Li 1994; Rodrigues \& da Silva 2007). Consequentemente, é possível usar um gerador de Box-Müller (Billinton \& Li 1994) para sortear o pico de carga para cada estado do sistema selecionado pelo MMC:

$$
L^{i}=L^{o}+L^{o}\left(\frac{\sigma^{o}}{100}\right) X^{n o r m}
$$

onde $L^{i}$ é o pico de carga do sistema para o estado i, $L^{o}$ é o pico de carga do sistema para a condição do caso-base, $\sigma^{\circ}$ é o erro de previsão de carga (desvio padrão) expresso como uma porcentagem de $L^{o}$ e $X^{\text {norm }}$ é um número aleatório com distribuição normal obtido através do gerador de BoxMüller. Por outro lado, a indisponibilidade dos equipamentos foi modelada considerando que as falhas dos componentes são independentes e representadas por um modelo de dois estados. Consequentemente, a probabilidade de falha é igual à indisponibilidade não-programada do equipamento. Desta forma, o estado de um equipamento é amostrado da seguinte forma:

i) Gerar um número aleatório $X^{\text {unif }}$ com distribuição uniforme entre 0 e 1 ;

ii) ii) $s_{j}^{i}= \begin{cases}1 \text { (estado operativo), } & \text { se } X^{\text {unif }}>U_{j} \\ 0 \text { (estado falhado), } & \text { se } 0 \leq X^{\text {unif }} \leq U_{j}\end{cases}$ onde $s_{j}^{i}$ é o estado do componente j no estado do sistema i e $U_{j}$ é a indisponibilidade não programada do componente j. Os estados dos componentes e o nível de carga são combinados para definir um estado do sistema da forma: $s^{i}=\left(s_{1}^{i}, s_{2}^{i}, \ldots, s_{N C}^{i}, L^{i}\right)$, onde $s^{i}$ é o i-ésimo estado do sistema e $N C$ é o número de componentes.

Os estados do sistema gerados pelo MMC podem apresentar perda de conectividade da rede (ilhamento) devido a falhas nos circuitos. Conseqüentemente, é necessário realizar um processamento topológico para identificar se o grafo da rede original foi particionado em subgrafos desconexos (ilhas). Neste artigo o processamento topológico é realizado usandose técnicas de varredura de grafos tais como, a busca em profundidade e em superfície descritas por Preiss (1999).

A geração de cada ilha, identificada no processamento topológico, deve ser despachada para satisfazer a sua carga. Em sistemas térmicos, o despacho é obtido usando-se uma lista de ordem de mérito dos custos incrementais de produção associados com os geradores. Por outro lado, em sistemas hidroelétricos não é possível associar custos de produção com os geradores. Uma alternativa para contornar esta dificuldade é realizar o despacho através da minimização do quadrado da distância Euclidiana (desvio quadrático) entre a potência de saída dos geradores e um padrão de geração especificado. Este padrão de geração é determinado pelo planejamento da operação de curto prazo para as usinas hidroelétricas (Oliveira, Secundino \& Nepomuceno 2005). É também possível utilizar pesos relativos para as usinas no despacho de geração baseado na minimização do desvio quadrático. Estes pesos definem o fator de participação de cada usina no despacho de geração em sistemas hidroelétricos. Além do despacho, pode ser necessário cortar carga para eliminar déficits de geração em alguns estados do sistema.

Após o despacho de carga/geração em cada ilha, é necessário compilar os dados necessários para realizar uma análise de fluxo de potência no estado amostrado. Esta compilação está associada com as seguintes tarefas: definição dos tipos das barras (PQ, PV e V $\theta$ ), cálculo dos limites MVAr em barras com geração de potência reativa, eliminação de barras isoladas e ilhas infactíveis (ilhas sem carga e geração), etc. É importante mencionar que a compilação de dados para o fluxo de potência define uma barra slack para cada subsistema isolado (ilha). Desta forma, é possível analisar um sistema composto por diversas ilhas com uma única análise de fluxo de potência.

O fluxo de potência é o ponto de partida para a análise de estabilidade de tensão de um estado amostrado. Se o algoritmo de fluxo de potência converge para uma solução, então o MMD é usado para determinar se o estado atual tem problemas de instabilidade de tensão causados pela perda de controlabilidade. Caso contrário, a Restauração da Solubilidade das Equações de Fluxo de Potência (RESFLUP) é realizada usando um fluxo de potência ótimo não-linear. Esta restauração é realizada para analisar a severidade dos estados de instabilidade de tensão associados com a ausência de solubilidade. Esta informação é muito importante para os operadores do sistema, pois permite identificar se há estados nos quais a insolubilidade pode ser eliminada sem usar o corte de carga. 
A amostra de estados do sistema avaliada com o MMD e pelo algoritmo de RESFLUP pode ser utilizada para estimar índices probabilísticos. Estes índices são calculados através da definição de média amostral:

$$
\tilde{E}(F)=\frac{1}{N S} \sum_{i=1}^{N S} F\left(s^{i}\right)
$$

onde:

NS é o número de estados do sistema,

$\tilde{E}(F)$ é a estimativa do valor esperado da função-teste.

$F\left(s^{i}\right)$ é o valor da função de teste associada com o índice $F$ no estado $s^{i}$. Por exemplo, se o índice estimado é a probabilidade de perda de carga, então $F\left(s^{i}\right)=1$ quando $s^{i}$ é um estado de falha (se há corte de carga no estado $s^{i}$ ), e $F\left(s^{i}\right)=$ 0 caso contrário.

A incerteza da estimativa $\tilde{E}(F)$ obtida com o MMC pode ser avaliada através do coeficiente de variação (Billinton \& Li 1994). Este coeficiente é dado por:

$$
\beta(F)=\frac{\tilde{\sigma}(F)}{\tilde{E}(F)}
$$

onde:

$\tilde{\sigma}(F)=\sqrt{\tilde{V} a r(F) / N S}$

$\tilde{V} \operatorname{ar}(F)=\frac{1}{N S-1} \sum_{i=1}^{N S}\left[F\left(s^{i}\right)-\tilde{E}(F)\right]^{2}$

$\tilde{V} \operatorname{ar}(F)$ é a variância amostral da função-teste,

$\tilde{\sigma}(F)$ é o desvio padrão amostral da função-teste.

Neste artigo, o coeficiente de variação é usado como regra de parada para o MMC. Entretanto, o número máximo de simulações é usado com um critério de retaguarda para evitar que um número excessivo de simulações seja realizado quando a precisão especificada é muito pequena.

\section{ANÁLISE DE ESTABILIDADE DE TEN- SÃO DE UM ESTADO DO SISTEMA}

\subsection{MMD}

Neste artigo o MMD (Prada et al. 2002) é usado para identificar se um estado tem problemas de instabilidade de tensão causados pela perda de controlabilidade. Este método se ba- seia no seguinte sistema linearizado das equações de fluxo de potência:

$$
\left[\begin{array}{c}
\Delta P \\
\Delta Q
\end{array}\right]=[J]\left[\begin{array}{c}
\Delta \theta \\
\Delta V
\end{array}\right]
$$

Colocando-se as equações referentes a barra $i$ em análise na parte inferior da equação (4) tem-se:

$$
\left[\begin{array}{c}
\Delta P^{\prime} \\
\Delta Q^{\prime} \\
\Delta P_{i} \\
\Delta Q_{i}
\end{array}\right]=\left[\begin{array}{cc}
A & B \\
C & D
\end{array}\right]\left[\begin{array}{c}
\Delta \theta^{\prime} \\
\Delta V^{\prime} \\
\Delta \theta_{i} \\
\Delta V_{i}
\end{array}\right]
$$

onde as submatrizes $A, B, C$ e $D$ são originadas a partir da partição da matriz Jacobiana completa $[J]$. Assumindo uma variação incremental $\Delta P$ e $\Delta Q$ apenas na barra i $\left(\Delta P^{\prime}=\Delta Q^{\prime}=0\right)$, é possível eliminar o acoplamento entre $\left[\begin{array}{ll}\Delta \theta & \Delta V\end{array}\right]$ e $\left[\Delta \theta^{\prime} \Delta V^{\prime}\right]$ através de uma redução de Kron. Aplicando-se este procedimento na equação (5) tem-se:

$$
\left[\begin{array}{c}
\Delta P_{i} \\
\Delta Q_{i}
\end{array}\right]=\left[D^{\prime}\right]\left[\begin{array}{c}
\Delta \theta_{i} \\
\Delta V_{i}
\end{array}\right]
$$

onde $\left[D^{\prime}\right]_{(2 \times 2)}=[D]-[C]^{*}[A]^{-1 *[B]}$

A equação (6) expressa as relações de sensibilidade entre as injeções de potência e as tensões na barra i considerando o restante do sistema. A partir do determinante da matriz $D^{\prime}$ pode-se concluir que (Prada et al. 2002):

i) $\operatorname{det}\left[D^{\prime}\right]>$ 0: a barra i está operando na região estável da curva PV (metade superior);

ii) $\operatorname{det}\left[D^{\prime}\right]<0$ : a barra i está operando na região instável da curva PV (metade inferior), isto é, a barra i tem problemas de perda de controlabilidade.

iii) $\operatorname{det}\left[D^{\prime}\right]=0$ : a barra i está operando no ponto de máximo carregamento (ponta da curva).

\subsection{FPO Não-Linear}

Neste artigo a RESFLUP é realizada através da minimização do corte de carga nas barras sujeita às seguintes restrições: equações de balanço de potência ativa e reativa, limites sobre as gerações de potência ativa e reativa e intervalos especificados para as tensões nas barras com geração de potência reativa. Neste problema de fluxo de potência ótimo não-linear, as seguintes ações de controle tem sido usadas na RESFLUP: redespacho de geração de potência ativa, ajustes 
nas tensões terminais de barras com geração de potência reativa e corte de carga (como último recurso). O problema de FPO associado com a RESFLUP foi resolvido usando o algoritmo de Pontos-Interiores proposto por Granville, Mello \& Melo (1996).

A principal vantagem de usar o FPO não-linear para realizar a RESFLUP é a capacidade desta técnica para identificar se é necessário realizar corte de carga para eliminar a insolubilidade das equações de fluxo de potência. Esta identificação não é possível quando a RESFLUP se baseia no Fluxo de Potência Continuado (Ajjarapu \& Christy 1992), pois a carga e a geração são simultaneamente reduzidas ou aumentadas em cada passo previsor/corretor.

\section{4 ÍNDICES PROPOSTOS PARA A APET}

O principal índice usado na APET é o RIT. O RIT expressa a probabilidade de ocorrência de estados do sistema com problemas de instabilidade de tensão. Neste artigo um estado instável é definido como aquele onde as equações de fluxo de potência não tem solução (ausência de solubilidade) ou $\Lambda_{\text {min }}^{j} \leq 0$ (perda de controlabilidade), onde $\Lambda_{\min }^{j}$ é o valor mínimo de $\operatorname{det}\left[D^{\prime}\right]$ para todas as barras PQ e PV no estado j do sistema. Além do RIT, a APET proposta neste artigo também se baseia na probabilidade dos estados de robustez (Fotuhi-Firuzabad \& Billinton 1997; Leite da Silva et al. 2008). A análise de robustez tem como objetivo estabelecer um elo entre a análise probabilística e a operação de sistemas de potência, que é tradicionalmente dominada por critérios determinísticos tais como o N-1. Este elo se baseia na definição de estados de robustez, que são similares aos estados usados na análise de segurança de sistemas de potência. Neste artigo o critério utilizado para definir os estados de robustez foi a ocorrência de problemas de instabilidade de tensão. Este critério foi selecionado devido a sua utilização em estudos de segurança de tensão realizados pelo Operador Nacional do Sistema Elétrico (2007). Usando-se este critério é possível definir os seguintes estados de robustez:

i) Estado Saudável: as equações de fluxo de potência têm uma solução e $\Lambda_{\min }^{j}>0$;

ii) Estado Marginal: as equações de fluxo de potência têm uma solução e $\Lambda_{\min }^{j} \leq 0$;

iii) Estado de Emergência: as equações de fluxo de potência não têm uma solução, mas é possível realizar a RESFLUP sem usar corte de carga;

iv) Estado Colapso: as equações de fluxo de potência não têm solução e só é possível restaurá-la com corte de carga.

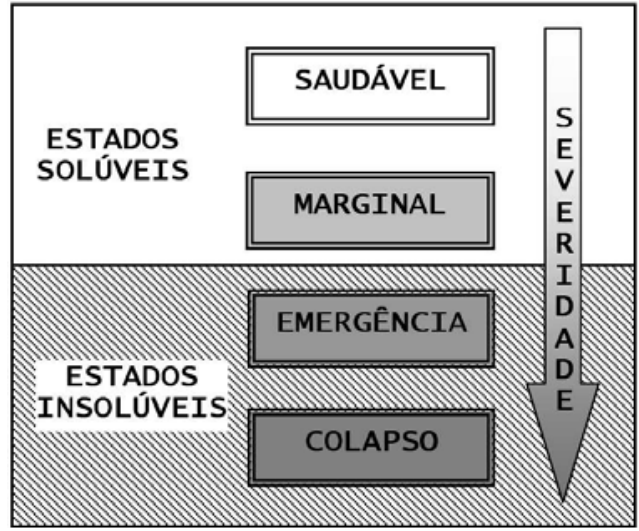

Figura 1: Estados de Robustez usados na APET

A partir das definições dos estados de robustez pode-se concluir que:

i) Os problemas de instabilidade de tensão associados com a ausência de solubilidade são considerados mais severos do que aqueles causados pela perda de controlabilidade.

ii) Os estados de robustez são definidos de acordo com a severidade dos mecanismos causadores da instabilidade de tensão, isto é: ausência de solubilidade e perda de controlabilidade. Na Figura 1 mostra-se os estados de robustez em ordem crescente de severidade.

Finalmente, o modelo proposto para a APET também foi usado para estimar o valor esperado da MET nodal. Neste artigo, o valor esperado da MET é calculado considerandose somente os estados onde as equações de fluxo de potência têm solução. Esta restrição é devido aos estados insolúveis serem considerados mais severos do que os estados com perda de controlabilidade. Devido a isto, não faz sentido calcular o valor esperado da MET nodal incluindo estados com ausência de solubilidade.

\section{RESULTADOS DOS TESTES}

O método proposto para a APET foi testado nos seguintes sistemas-teste: dois sistemas equivalentes obtidos a partir do Sistema Interligado Nacional (Alves 2007) e uma versão modificada do sistema IEEE para estudos de confiabilidade (Bertoldi, Salvaderi \& Scalcino 1988). As principais características dos sistemas teste são apresentadas na Tabela 1. Nesta tabela, as siglas MRTS, STB-65 e STB-107 estão associadas com os seguintes sistemas-teste: versão modificada do sistema IEEE para estudos de confiabilidade, sistema brasileiro de 65 barras e sistema brasileiro de 107 barras, respectivamente. Os dados de confiabilidade dos sistemas STB-65 
e STB-107 foram obtidos de (Schilling, Stacchini de Souza $\&$ Do Couto Filho 2008).

Tabela 1: Características dos sistemas-teste

\begin{tabular}{|c|c|c|c|}
\hline \multirow{2}{*}{ Característica } & \multicolumn{3}{|c|}{ Sistemas } \\
\hline & MRTS & STB-65 & $\begin{array}{l}\text { STB- } \\
107\end{array}$ \\
\hline $\begin{array}{c}\text { Capacidade instalada } \\
\text { (MW) }\end{array}$ & 4304,0 & 17858,2 & 22080,2 \\
\hline Pico de carga (MW) & 3562,4 & 10102,1 & 12681,7 \\
\hline $\mathrm{N}^{o}$ de barras & 24 & 65 & 107 \\
\hline $\mathrm{N}^{o}$ de circuitos & 31 & 141 & 230 \\
\hline $\mathrm{N}^{o}$ de geradores & 40 & 65 & 103 \\
\hline $\mathrm{N}^{o}$ de usinas & 10 & 14 & 23 \\
\hline $\begin{array}{c}\mathrm{N}^{o} \text { de } \\
\text { compensadores }\end{array}$ & 1 & 4 & 5 \\
\hline
\end{tabular}

Os índices probabilísticos associados com os sistemas-teste foram estimados considerando-se que:

i) O erro de previsão de carga $\left(\sigma^{\circ}\right)$ é 5,0\%;

ii) A APET foi realizada considerando-se contingências em circuitos, geradores e compensadores;

iii) A tolerância pré-especificada para o coeficiente de variação é 5,0\%;

iv) O número máximo de simulações é 100.000 para o sistema MRTS e 50.000 para os sistemas brasileiros.

v) Os pesos relativos, usados no despacho de geração, de todas as usinas dos sistemas brasileiros são unitários.

vi) O coeficiente de variação foi usado para a convergência de todos os índices estimados: RIT e probabilidades dos estados de robustez.

O esforço computacional (número de simulações e tempo de processamento) requerido para estimar os índices probabilísticos de acordo com as condições (i)-(v) é mostrado na Tabela 2. Os resultados mostrados nesta tabela foram obtidos usando-se um PC com processador Intel Core Quad de 2,4 GHz e 3,25 GB de RAM. A partir da Tabela 2, pode-se concluir que o número de simulações, exigido para satisfazer a tolerância especificada (5\%), não é dependente do tamanho do sistema. De fato, o número de simulações no MMC dependente apenas dos seguintes parâmetros do índice estimado: variância, valor esperado e tolerância especificada (Billinton \& Li 1994).
O principal índice usado na APET é o RIT. Os valores do RIT para os sistemas MRTS, STB-65 e STB-107 são iguais a: $26,3484 \%, 28,7540 \%$ e $26,2045 \%$, respectivamente. Desta forma, pode-se concluir que o RIT é bastante elevado para os três sistemas-teste. Entretanto, as causas dos valores altos do RIT são distintas para os três sistemas. Este fato pode ser demonstrado através do cálculo das porcentagens das incertezas no RIT. Estas são mostradas na Tabela 3. Nesta tabela as siglas G, R, C e GR estão associadas com os seguintes eventos: falhas de geradores, contingências em ramos (linhas e transformadores), flutuações no pico de carga devido a erros de previsão (sem equipamentos fora de serviço) e falhas simultâneas de geradores e ramos, respectivamente. A partir da Tabela 3 pode-se concluir que:

i) Os estados instáveis dos sistemas MRTS e STB-107 são causados principalmente por falhas nos geradores. Este resultado pode ser um indicativo da existência de deficiências na reserva de geração de potência reativa nos sistemas MRTS e STB-107.

ii) No sistema STB-65, a principal causa de problemas de instabilidade de tensão é o erro de previsão de carga. Este efeito revela que o ponto de operação do STB-65 é de carga pesada com a rede bem carregada.

iii) As porcentagens associadas com o erro de previsão de carga são significantes para os três sistemas-teste. Por exemplo, o valor mínimo da porcentagem é 20,3056 para o sistema STB-107.

iv) $\mathrm{O}$ sistema onde as indisponibilidades dos circuitos têm maior impacto no RIT é o STB-65. Desta forma, pode ser necessário realizar reforços na rede de transmissão do STB-65.

Tabela 2: Esforço computacional para realizar a APET nos sistemas teste

\begin{tabular}{|c|c|c|}
\hline Sistema & $\begin{array}{c}\mathbf{N}^{o} \text { de } \\
\text { simulações }\end{array}$ & $\begin{array}{c}\text { Tempo de } \\
\text { processamento } \\
\text { (minutos) }\end{array}$ \\
\hline MRTS & 42.401 & 4,5036 \\
\hline STB-65 & 38.193 & 8,0242 \\
\hline STB-107 & 39.955 & 25,2453 \\
\hline
\end{tabular}

A APET dos sistemas-teste também foi realizada usando-se índices de robustez. Estes índices permitem realizar uma análise da estabilidade de tensão baseada nos mecanismos causadores da instabilidade. As probabilidades dos estados de robustez são apresentadas na Tabela 4 . As probabilidades 
mostradas nesta tabela também podem ser usadas para estimar as porcentagens dos estados de robustez instáveis (marginal, emergência e colapso) no RIT. Estas porcentagens são mostradas na Tabela 5.

Tabela 3: Porcentagem das incertezas no RIT

\begin{tabular}{|c|c|c|c|}
\hline Incertezas & MRTS & STB-65 & STB-107 \\
\hline G & 69,8980 & 19,1950 & 42,0535 \\
\hline R & 0,9846 & 24,2488 & 13,1614 \\
\hline GR & 4,2338 & 9,7159 & 24,4795 \\
\hline C & 24,8836 & 46,8403 & 20,3056 \\
\hline
\end{tabular}

Tabela 4: Probabilidades dos estados de robustez

\begin{tabular}{|c|c|c|c|}
\hline Estados & MRTS & STB-65 & STB-107 \\
\hline Saudável & $7,3652 \times 10^{-1}$ & $7,1246 \times 10^{-1}$ & $7,3796 \times 10^{-1}$ \\
\hline Marginal & $9,3630 \times 10^{-3}$ & $2,3986 \times 10^{-1}$ & $9,9362 \times 10^{-3}$ \\
\hline Emergência & $2,4077 \times 10^{-1}$ & $3,7311 \times 10^{-2}$ & $2,3919 \times 10^{-1}$ \\
\hline Colapso & $1,3349 \times 10^{-2}$ & $1,0368 \times 10^{-2}$ & $1,2915 \times 10^{-2}$ \\
\hline
\end{tabular}

Tabela 5: Porcentagens dos estados de robustez com relação ao RIT

\begin{tabular}{|c|c|c|c|}
\hline Estados & MRTS & STB-65 & STB-107 \\
\hline Marginal & $3,5535 \%$ & $83,4183 \%$ & $3,7918 \%$ \\
\hline Emergência & $91,3802 \%$ & $12,9758 \%$ & $91,2798 \%$ \\
\hline Colapso & $5,0662 \%$ & $3,6059 \%$ & $4,9284 \%$ \\
\hline
\end{tabular}

A partir das Tabelas 4 e 5 pode-se concluir que:

i) O estado de emergência é o mais provável dos estados nos sistemas MRTS e STB-107. Em outras palavras, nestes sistemas a principal causa da instabilidade de tensão é a insolubilidade das equações de fluxo de potência.

ii) O valor máximo da probabilidade de ocorrência do estado marginal está associado com o sistema STB-65. Devido a isto, o maior valor da porcentagem do estado marginal, com relação ao RIT, ocorre no STB-65 $(83,4183 \%)$. Isto é, no sistema STB-65 os estados instáveis são causados principalmente pela perda de controlabilidade.

iii) As porcentagens do estado de colapso, com relação ao RIT, são pequenas para os três sistemas. Por exemplo, o valor máximo desta porcentagem para os três sistemas é 5,0662\%. Consequentemente, é possível restaurar a solução das equações de fluxo de potência na maioria dos estados insolúveis sem realizar corte de carga.

As probabilidades dos estados de robustez também permitem avaliar a eficiência dos controles usados na RESFLUP. Esta avaliação é realizada calculando-se as porcentagens das probabilidades dos estados de robustez insolúveis (Emergência e Colapso) com relação à probabilidade de insolubilidade. Estas porcentagens são mostradas na Tabela 6.

Tabela 6: Eficiência das ações de controle usadas na RESFLUP

\begin{tabular}{|c|c|c|c|}
\hline Estado & MRTS & STB-65 & STB-107 \\
\hline Emergência & $94,7471 \%$ & $78,2537 \%$ & $94,8774 \%$ \\
\hline Colapso & $5,2529 \%$ & $21,7463 \%$ & $5,1226 \%$ \\
\hline
\end{tabular}

A partir da Tabela 6, pode-se concluir que o redespacho da geração e o ajuste das tensões em barras $\mathrm{PV}$ e $\mathrm{V} \theta$ foram altamente eficientes para eliminar problemas de insolubilidade nos sistemas MRTS e STB-107. Por exemplo, o valor mínimo da porcentagem associada com o estado de emergência nestes sistemas é $94,7471 \% \%$. Isto é, a porcentagem máxima de estados que exigem corte de carga na RESFLUP é $5,2529 \%$. Por outro lado, as ações corretivas usadas na RESFLUP obtiveram uma eficiência de apenas $78,2537 \%$ no STB-65. Consequentemente, a ausência de solubilidade é mais severa naqueles sistemas.

Adicionalmente, deve-se mencionar que há uma probabilidade de ocorrer perda de controlabilidade após a RESFLUP. Ou seja, a solução obtida pelo algoritmo de RESFLUP está localizada na região instável (metade inferior) da curva PV. As probabilidades de um estado ter problemas de insolubilidade e perda de controlabilidade para os sistemas MRTS, STB-65 e STB-107 são iguais a: 0,0684\%, 4,5977\% e 4,5376\%, respectivamente. Estes resultados demonstram que a probabilidade de ocorrência dos dois mecanismos causadores da instabilidade é mais significativa nos sistemas brasileiros. Este fato é devido à ausência de restrições de estabilidade de tensão no algoritmo de FPO usado para realizar a RESFLUP.

\section{CONCLUSÕES}

Descreveu-se um método para incluir insolubilidade e perda de controlabilidade na APET (Análise Probabilística da Estabilidade de Tensão). Este método é baseado na combinação de três técnicas: MMD (Método da Matriz D'), FPO (Fluxo de Potência Ótimo) não-linear e MMC (Método Monte Carlo). Os resultados obtidos com a técnica proposta nos sistemas-teste demonstraram que: 
i) Erros de previsão de carga tem uma porcentagem significante no RIT (Risco de Instabilidade de Tensão).

ii) Estados instáveis associados com a perda de controlabilidade têm uma probabilidade de ocorrência considerável.

iii) A RESFLUP (Restauração da Solubilidade das Equações de Fluxo de Potência) pode resultar em estados com perda de controlabilidade.

iv) As ações de controle usadas na RESFLUP foram bastante eficientes na maioria dos sistemas-teste.

As principais contribuições do método de APET com relação às técnicas existentes são: a modelagem dos dois mecanismos causadores da instabilidade de tensão (a perda de controlabilidade e a insolubilidade) no cálculo do RIT, a avaliação da severidade dos estados de instabilidade de tensão através da definição de índices de robustez e a modelagem dos principais distúrbios causadores de problemas de colapso de tensão (falhas nos equipamentos e flutuações no pico de carga devido a erros de previsão).

\section{REFERÊNCIAS}

Aboreshaid, S. \& Billinton, R. (1999) Probabilistic Evaluation of Voltage Stability. IEEE Trans. Power System, 14 (1) Fevereiro, pp. 342-348.

Alves, W. F. (2007) Proposição de sistemas-teste para análise computacional de sistemas de potência. Dissertação de Mestrado, Universidade Federal Fluminense.

Ajjarapu, V. \& Christy, C. (1992) The Continuation Power Flow: A Tool for Steady State Voltage Stability Analysis. IEEE Trans. Power System, 7 (1) Fevereiro, pp. 416-422.

Ajjarapu, V. (2006) Computational Techniques for Voltage Stability Assessment and Control. Springer.

Bertoldi, O., Salvaderi, L., \& Scalcino, S. (1988) Monte Carlo Approach in Planning Studies - An Application to IEEE RTS. IEEE Trans. Power System, 3 (3) Agosto, pp. 1146-1154.

Billinton, R. \& Li, W. (1994) Reliability Assessment of Electric Power Systems Using Monte Carlo Methods. Plenum Press.

Billinton, R. \& Allan, R. N. (1996) Reliability Evaluation of Power Systems. 2nd ed. Plenum Press.

Billinton, R. \& Aboreshaid, S. (1998) Voltage Stability Considerations in Composite Power System Reliability Evaluation. IEEE Trans. Power Systems, 13 (2) Maio, pp. 655-660.
Fotuhi-Firuzabad, M. \& Billinton, R. (1997) A energy Base Approach to Evaluate Interruptible Load Carrying Capability in Isolated and Interconnected Systems Including Well-Being Constraints. IEEE Trans. Power Systems, 12 (4) Maio, pp. 1676-1681.

Gao, B., Morison, G. K. \& Kundur, P. (1992) Voltage stability evaluation using modal analysis. IEEE Trans. Power Systems, 7 (4) Novembro, pp. 1529-1542.

Granville, S., Mello, J. C. O., \& Melo, A. C. G. (1996) Application of Interior Point Methods to Power Flow Unsolvability. IEEE Trans. on Power System, 11 (2) Maio, pp. 1096-1103.

Huang, G. M. \& Nair, N. C. (2002) Voltage Stability Constrained Load Curtailment Procedure to Evaluate Power System Reliability Measures. Em: IEEE Power Engineering Society Winter Meeting, January 27-31, 2002, New York USA, pp. 761-765.

Kundur, P. (1994) Power System Stability and Control. McGraw-Hill.

Leite da Silva, A. M., Coutinho, I. P., Zambroni de Souza, A. C., Prada, R. B., \& Rei, A. M. (2000) Voltage Collapse Risk Assessment. Electric Power System Research, 54 (3) Junho, pp. 221-227.

Leite da Silva, A. M., Resende, L. C., Manso, L. A. F., \& Miranda, V. (2008) Well-being analysis for composite generation and transmission systems based on pattern recognition techniques. IET Gener. Transm. Distrib., 2 (2) Março, pp. 202-208.

Li, W., Mansour, Y., Vaahedi, E., \& Pettet, D. N. (1998) Incorporatiing of Voltage Stability Operation Limits in Composite Systems Adequacy Assessment: BC Hydro's Experience. IEEE Trans. Power System, 13 (4) Novembro, pp. 1279-1284.

Melo, A. C. G., Mello, J. C. O. \& Granville, S. (1997) The Effect of Voltage Collapse Problems in the Reliability Evaluation of Composite Systems. IEEE Trans. Power Systems, 12 (1) Fevereiro, pp. 480-488.

Oliveira, A. R. L., Soares, S. \& Nepomuceno, L. (2005) Short term hydroelectric scheduling combining network flow and interior-point approaches. International Journal of Electrical Power \& Energy Systems, 27 (2) Fevereiro, pp. 91-99.

Operador Nacional do Sistema Elétrico (2007) Diretrizes e Critérios para Estudos Elétricos-Procedimentos de Rede-Submódulo 23.3 [Internet], Disponível de: <http://www.ons.org.br> [Acessado em 18 de agosto de 2009] 
Overbye, T. J. (1994) A Power Flow Measure for Unsolvable Cases. IEEE Trans. on Power Systems, 9 (3) Agosto, pp. 1359-1365.

Prada, R. B., Palomino, E. G. C., dos Santos, J. O. R., \& Pilloto, L. A. S. (2002) Voltage Stability Assessment for Real-Time Operation. IEE-Proc.-Gener. Transm. Distrib., 149 (2) Março, pp. 175-181.

Preiss, B. R (1999) Data Structures and Algorithms with Object-Oriented Design Patterns in C++. John Wiley \& Sons.

Rodrigues, A. B. \& da Silva, M. G. (2007) Probabilistic Assessment of Available Transfer Capability Based on Monte Carlo Method with Sequential Simulation. IEEE Trans. Power Systems, Vol. 22, No. 1; pp. 484-492.

Schilling, M. T., Stacchini de Souza, J. C. \& do Couto Filho, M. B. (2008) Power System Probabilistic Reliability Assessment: Current Procedures in Brazil. IEEE Trans. Power Systems, 23 (3) Agosto, pp. 868-876.

Wan, H., McCalley, J. D. \& Vittal, V. (2000) Risk Based Voltage Security Assessment. IEEE Trans. Power Systems, 15 (4) Novembro, pp. 1247-1254. 